ARTICLE

\title{
Amorphization-induced surface electronic states modulation of cobaltous oxide nanosheets for lithium-sulfur batteries
}

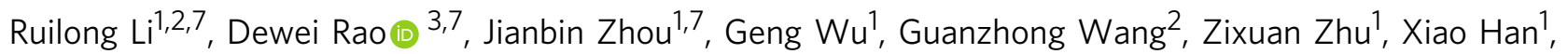
Rongbo Sun ${ }^{1}$, Hai Li (iD ${ }^{4}$, Chao Wang ${ }^{5}$, Wensheng Yan (1) ${ }^{5}$, Xusheng Zheng ${ }^{5}$, Peixin Cui (i) ${ }^{6}$, Yuen Wu (1) ${ }^{1}$, Gongming Wang (i) ${ }^{1 凶} \&$ Xun Hong (i) ${ }^{1 凶}$

Lithium-sulfur batteries show great potential to achieve high-energy-density storage, but their long-term stability is still limited due to the shuttle effect caused by the dissolution of polysulfides into electrolyte. Herein, we report a strategy of significantly improving the polysulfides adsorption capability of cobaltous oxide by amorphization-induced surface electronic states modulation. The amorphous cobaltous oxide nanosheets as the cathode additives for lithium-sulfur batteries demonstrates the rate capability and cycling stability with an initial capacity of $1248.2 \mathrm{mAh} \mathrm{g}^{-1}$ at $1 \mathrm{C}$ and a substantial capacity retention of $1037.3 \mathrm{mAh} \mathrm{g}^{-1}$ after 500 cycles. X-ray absorption spectroscopy analysis reveal that the coordination structures and symmetry of ligand field around Co atoms of cobaltous oxide nanosheets are notably changed after amorphization. Moreover, DFT studies further indicate that amorphization-induced re-distribution of $d$ orbital makes more electrons occupy high energy level, thereby resulting in a high binding energy with polysulfides for favorable adsorption.

\footnotetext{
${ }^{1}$ Center of Advanced Nanocatalysis (CAN), Hefei National Laboratory for Physical Sciences at the Microscale, Department of Applied Chemistry, University of Science and Technology of China, Hefei, Anhui, China. ${ }^{2}$ Department of Physics, University of Science and Technology of China, Hefei, Anhui, China.

${ }^{3}$ School of Materials Science and Engineering, Jiangsu University, Zhenjiang, Jiangsu, China. ${ }^{4}$ Key Laboratory of Flexible Electronics (KLOFE) \& Institute of Advanced Materials (IAM), Jiangsu National Synergetic Innovation Center for Advanced Materials (SICAM), Nanjing Technology University, Nanjing, Jiangsu, China. ${ }^{5}$ National Synchrotron Radiation Laboratory (NSRL), University of Science and Technology of China, Hefei, Anhui, P.R. China. ${ }^{6}$ Key Laboratory of Soil Environment and Pollution Remediation, Institute of Soil Science, Chinese Academy of Sciences, Nanjing, P.R. China. ${ }^{7}$ These authors contributed

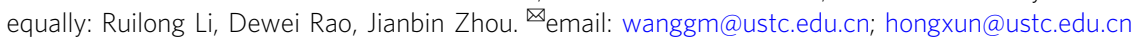


D espite the high theoretical energy density and capacity of $\mathrm{Li}-\mathrm{S}$ batteries, their practical applications are limited by the shuttle effect, which is originated from the dissolution of lithium polysulfides $\left(\mathrm{Li}_{2} \mathrm{~S}_{x}, x \geq 4\right.$, LiPSs) in electrolyte ${ }^{1-3}$. To suppress the shuttle effect, significant efforts have been made in utilizing host materials to adsorb LiPSs via chemical interactions such as polar interactions, Lewis acid-base interactions, and catenation ${ }^{4-6}$. For example, metal oxides such as $\mathrm{TiO}_{2}, \mathrm{Co}_{3} \mathrm{O}_{4}$, $\mathrm{MnO}_{2}$, and $\mathrm{V}_{2} \mathrm{O}_{5}{ }^{7-10}$, have been demonstrated as efficient sulfur host materials for anchoring LiPSs with the binding energy of usually $2.6-3.5 \mathrm{eV}^{11}$. Compared with the above values, higher binding energy tends to be favorable to promote kinetics and suppress capacity fading in Li-S batteries ${ }^{12,13}$, therefore, further enhancement of the LiPSs adsorption capability remains an urgent need.

The adsorption of LiPSs has closely related to the surface electronic states of the substrates ${ }^{14}$. Moreover, ultrathin nanosheets are considered to possess excellent structures to offer abundant binding sites with LiPSs ${ }^{15}$. Thus, the rational modulation of surface electronic states of ultrathin structures to enhance the binding energy with LiPSs is available but still remains a substantial challenge owing to the elusive adsorption mechanism ${ }^{16-19}$. Recently, amorphous materials, with a special regulation of atomic arrangement, provide the possibility for fine-tuning of electronic properties towards favorable electrochemical intermediates adsorption ${ }^{20,21}$. Because of the broken atomic long-range order, amorphous materials present unique surface electronic states 22 , which may contribute to further enhancement of the LiPSs adsorption. Therefore, combining the ultrathin structure with the amorphous structure to boost the LiPSs adsorption capability of metal oxides is highly desirable but challenging as well due to the difficulty of size and shape control of amorphous materials ${ }^{23,24}$.

Herein, ultrathin amorphous $\mathrm{CoO}$ nanosheets (a-CoO NSs) with a thickness of about $10 \mathrm{~nm}$ were synthesized and selected as the sulfur host. X-ray absorption spectroscopy analysis reveals that the a-CoO NSs possess abundant coordinatively unsaturated metal sites and the symmetry of ligand field around Co atoms partially convert from the octahedral $\left(O_{\mathrm{h}}\right)$ to the tetrahedral $\left(T_{\mathrm{d}}\right)$ configurations. Density functional theory (DFT) calculations reveal that the adjusted Co $d$ orbitals in a-CoO NSs result in a significantly higher binding energy toward $\mathrm{Li}_{2} \mathrm{~S}_{4}$ than that of the crystalline counterpart. Thus, the a-CoO NSs exhibits cycling stability with only $0.034 \%$ capacity decay per cycle for 500 cycles as the cathode additives for $\mathrm{Li}-\mathrm{S}$ batteries.

\section{Results}

Synthesis and characterization of a-CoO NSs. In a typical synthesis, cobalt(II) acetylacetonate $\left(\mathrm{Co}(\mathrm{acac})_{2}\right)$ and potassium bromide $(\mathrm{KBr})$ were firstly well dissolved in water/ethanol mixed solution and stirring for two hours. After the solution was evaporated at $80^{\circ} \mathrm{C}$, the dried mixture was transferred into a porcelain boat and annealed at $240{ }^{\circ} \mathrm{C}$ for $80 \mathrm{~min}$ under air atmosphere. Finally, after washing to remove $\mathrm{KBr}$ and centrifugation, the a-CoO NSs were obtained. Moreover, we utilize temperature control to regulate the atomic structure in $\mathrm{CoO}$ nanosheets, which leads to the formation of disordered atomic structure at a low temperature $\left(240{ }^{\circ} \mathrm{C}\right)$ and the crystalline counterpart (c-CoO NSs, Supplementary Fig. 1) with long-range ordered atomic structure at a higher temperature $\left(400^{\circ} \mathrm{C}\right)$.

In Fig. 1a, the X-ray diffraction (XRD) patterns demonstrate the crystallinity of a-CoO NSs and c-CoO NSs. The a-CoO NSs display no obvious peak, indicating the amorphous structure, while the c-CoO NSs exhibits sharp peaks indexed to the crystalline CoO (PDF\#48-1719, Supplementary Fig. 2). TEM (Fig. 1b) image confirmed the nanosheet morphology with the lateral size of micrometers of the a-CoO NSs. The AFM image and its height profile in Fig. 1c show the ultrathin 2D structure of a-CoO NSs with a thickness of about $10.4 \mathrm{~nm}$. High-angle annular dark-field (HAADF) images of scanning TEM (STEM) was further recorded to investigate the atomic structure of a-CoO NSs. As displayed in Fig. 1d, a-CoO NSs exhibit randomly distributed atoms with no continuous crystal lattice stripes, indicating the lack of long-range order of atomic structure. Moreover, the weak ring in the electron diffraction (ED) pattern of a-CoO NSs (Fig. 1e) further reveals its amorphous structure. HAADF-STEM images of a-CoO NSs and corresponding energydispersive X-ray spectroscopy (EDS) elemental mapping images (Fig. 1f) show that $\mathrm{Co}, \mathrm{O}$, and $\mathrm{C}$ elements are homogeneously distributed over the entire nanosheets.

Atomic and electronic structures of a-CoO NSs. Raman spectrum of the a-CoO NSs (Fig. 2a) show typical vibration peaks of $\mathrm{CoO}$, the peaks at 478,516 , and $680 \mathrm{~cm}^{-1}$ were identified as $E_{\mathrm{g}}$, $T_{2 \mathrm{~g}}$, and $A_{1 \mathrm{~g}}$ modes in $\mathrm{O}_{\mathrm{h}}$ symmetry, respectively ${ }^{25}$, which reveals that the $\mathrm{Co}-\mathrm{O}$ bonding mainly in the $\mathrm{CoO}_{6}$ octahedron motif. Compared with c-CoO NSs, a-CoO NSs shows widened and slightly blue-shifted Raman peaks owing to the lack of long-range order of $\mathrm{CoO}_{6}$ octahedra ${ }^{26}$. Moreover, the Co $2 p$ XPS spectra (Fig. 2b) further reveal that a-CoO NSs owns the characteristic peaks of Co (II) oxidation state located at 781.1 (Co 2p $p_{3 / 2}$ ) and $797.1 \mathrm{eV}\left(2 p_{1 / 2}\right)$, respectively, and two corresponding shake-up satellite peaks, which are similar with that of $\mathrm{c}-\mathrm{CoO} \mathrm{NSs}^{27}$. O $1 \mathrm{~s}$ XPS spectra (Supplementary Fig. 3) of both a-CoO NSs and cCoO NSs display a strong intense peak at $531.5 \mathrm{eV}$, which can be assigned to $\mathrm{Co}-\mathrm{O}$ bonding in $\mathrm{CoO}^{28}$.

X-ray absorption spectroscopy analysis was used to verify the electronic properties as well as coordination environment of the Co atoms in a-CoO NSs. The absorption edge position on X-ray absorption near-edge structure (XANES) spectra (Fig. 2c) of both a-CoO NSs and c-CoO NSs are located close to CoO, further suggesting the valence state of $\mathrm{Co}$ is +2 . The Fourier-transformed extended X-ray absorption fine structure (FT-EXAFS) spectra (Fig. 2d, Supplementary Fig. 4) of a-CoO NSs shows two main peaks corresponding to the $\mathrm{Co}-\mathrm{O}$ and $\mathrm{Co}-\mathrm{Co}$ coordination shells respectively. The fitted EXAFS structural parameters in Supplementary Table 1 show that the average coordination number of $\mathrm{Co}-\mathrm{O}$ shell $\left(\mathrm{N}_{\mathrm{Co}-\mathrm{O}}\right)$ in both a-CoO NSs $\left(\mathrm{N}_{\mathrm{Co}-\mathrm{O}}=5.5\right)$ and c-CoO NSs $\left(N_{\mathrm{Co}-\mathrm{O}}=5.8\right)$ are about 6 , which indicates that the two samples mainly own $\mathrm{CoO}_{6}$ octahedron units ${ }^{29}$. Moreover, the shortest $\mathrm{Co}-\mathrm{Co}$ distance of about $3.0 \AA$ indicates that both a-CoO NSs and c-CoO NSs own the edge-sharing connection mode between $\mathrm{CoO}_{6}$ octahedra ${ }^{30}$. The coordination number of $\mathrm{Co}-\mathrm{Co}$ shell $\left(N_{\mathrm{Co}}\right)$ of a-CoO NSs $\left(N_{\mathrm{Co}}=8.2\right)$ is lower than that of $\mathrm{c}-\mathrm{CoO}$ NSs $\left(N_{\mathrm{Co}}=10.1\right)$ and $\mathrm{CoO}\left(N_{\mathrm{Co}}=13.3\right)$. The reduced $N$ values of a-CoO NSs and c-CoO NSs derived from their low dimensional (2D) structures compared with $\mathrm{CoO}^{31}$. Moreover, the further reduced $\mathrm{N}$ value of a-CoO NSs derived from the low order within an extended structure of $\mathrm{CoO}_{6}$ octahedra caused by increased $\mathrm{CoO}_{6}$ octahedron vacancies ${ }^{32}$. Thus, the a-CoO NSs possess abundant coordinatively unsaturated metal sites ${ }^{33,34}$.

Co L-edge XANES were carried out to investigate the $3 d$ electronic configurations of $\mathrm{Co}^{2+}$ cations in the a-CoO NSs. The Co L-edge spectrum of the a-CoO NSs shows an obvious peak-shift $(0.3 \mathrm{eV})$ toward the low energy region, compared with that of $\mathrm{c}$ $\mathrm{CoO}$ NSs (Fig. 2e). Considering that the valence states of Co cations in both a-CoO NSs and c-CoO NSs are +2 , the negative peak shift can be attributed to the different symmetries of ligand field around $\mathrm{Co}^{2+}$ cations between a-CoO NSs and c-CoO NSs ${ }^{35,36}$. To examine the symmetry of ligand field around $\mathrm{Co}^{2+}$ cations in a-CoO NSs, simulations of the Co L-edge XANES spectra were performed by using CTM4XAS (Fig. 2f, Supplementary Tables 2 and 3 

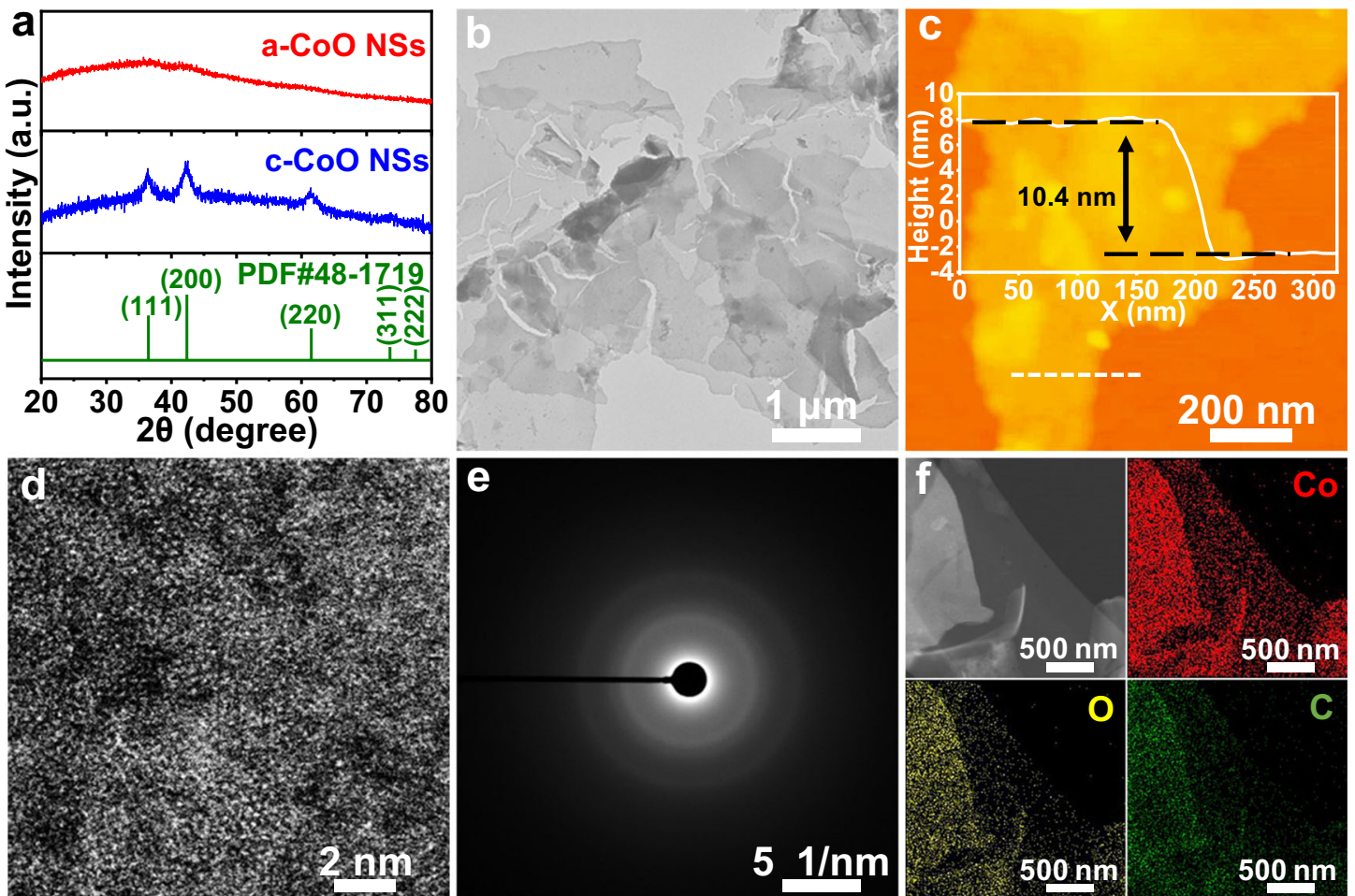

Fig. 1 Characterization of a-CoO NSs. a XRD patterns of a-CoO NSs and c-CoO NSs. b TEM, c AFM, and d HAADF-STEM images of a-CoO NSs. e ED pattern of a-CoO NSs. $\mathbf{f}$ HAADF-STEM image and corresponding EDS element mapping of a-CoO NSs: Co (red), O (yellow), and C (green).
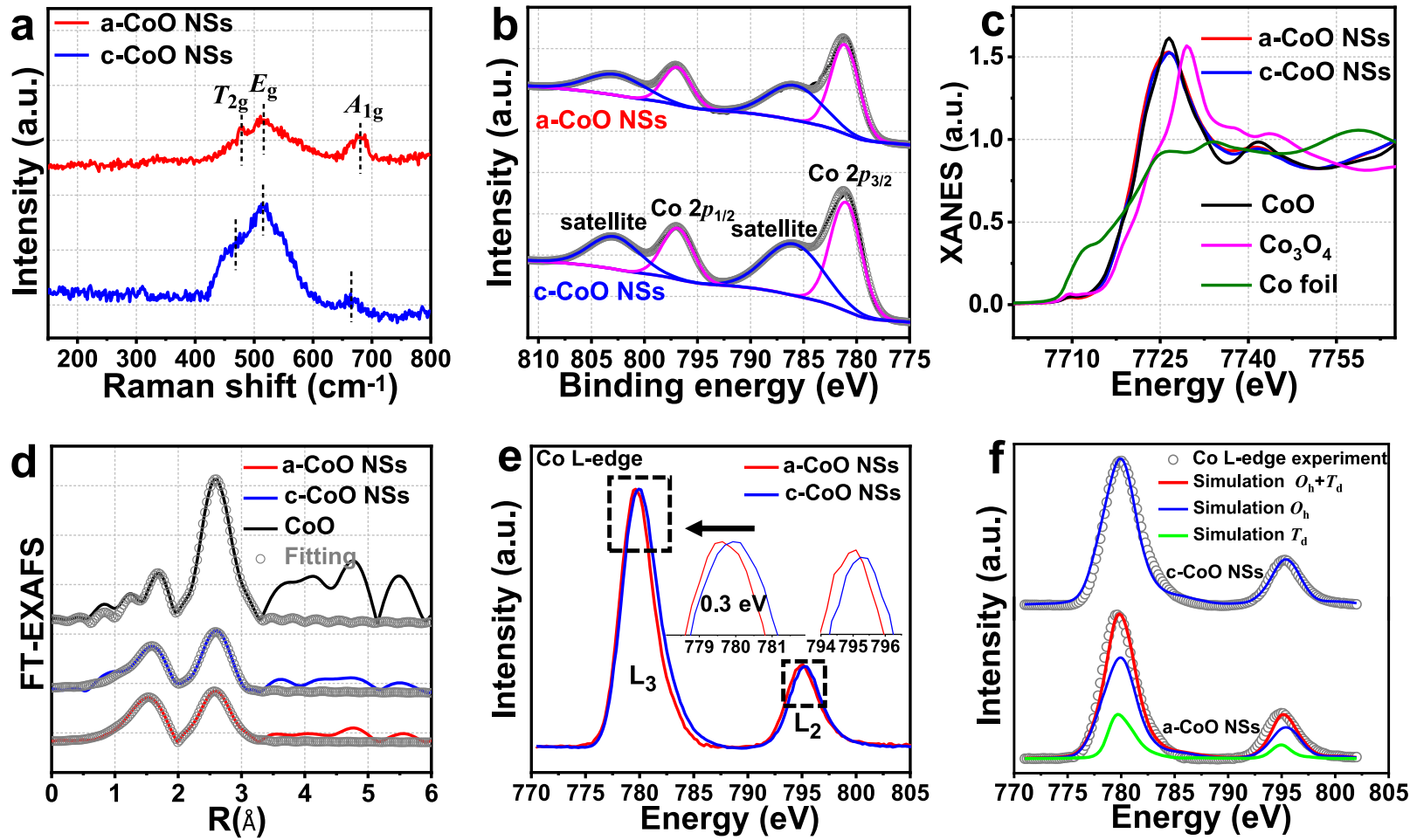

Fig. 2 Chemical states and coordination structure analysis. a Raman spectra of the a-CoO NSs and c-CoO NSs. b Co $2 p$ spectra of the a-CoO NSs and c$\mathrm{CoO} N S$ s. c XANES spectra at the $\mathrm{Co} \mathrm{K}$ edge of the a-CoO NSs, c-CoO NSs, $\mathrm{CoO}, \mathrm{Co}_{3} \mathrm{O}_{4}$ samples and Co foil. $\mathbf{d}$ Fourier transformed (FT)-EXAFS spectra of the a-CoO NSs, c-CoO NSs, and CoO. e Co L-edge XANES spectra (inset: the amplificatory image of $\mathrm{Co}_{0} \mathrm{~L}_{3}$ and $\mathrm{L}_{2}$ edge respectively) and $\mathbf{f}$ simulations of Co L-edge XANES spectra for the a-CoO NSs and c-CoO NSs. 

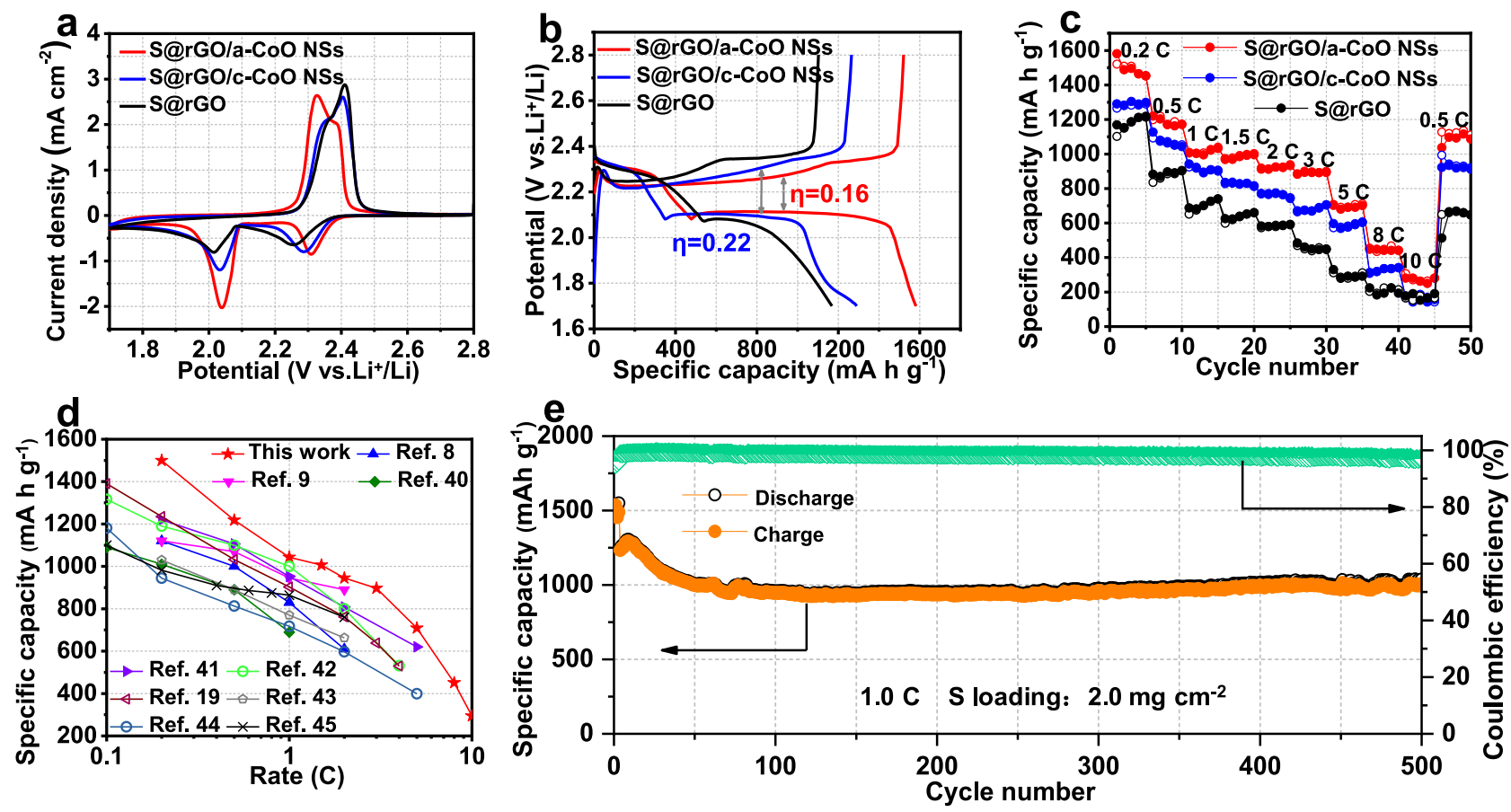

Fig. 3 Electrochemical studies toward Li-S batteries. a CV curves at a scan rate of $0.1 \mathrm{mV} \mathrm{s}^{-1}$ between 1.7 and $2.8 \mathrm{~V}$. b Galvanostatic charge-discharge profiles at 0.2 C. c Rate performance.d Comparison of the rate performance of the S@rGO/a-CoO NSs electrode in this work and other metal oxides-based electrodes recently reported. e Cycling performance at 1C of the S@rGO/a-CoO NSs electrode.

and "Methods"), in which the simulations of Co $\mathrm{L}_{3}$-edge spectra of $\mathrm{Co}^{2+}$ tetrahedral $\left(T_{\mathrm{d}}\right)$ sites and octahedral $\left(O_{\mathrm{h}}\right)$ sites are located at 779.7 and $780.0 \mathrm{eV}$, respectively (Supplementary Fig. 5a, b) ${ }^{37,38}$. As a result, Fig. $2 \mathrm{f}$ shows that the a-CoO NSs possess a mixture of $\mathrm{Co}^{2+} T_{\mathrm{d}}$ sites and $\mathrm{Co}^{2+} \mathrm{O}_{\mathrm{h}}$ sites with a ratio of $1: 2.3$, while the c$\mathrm{CoO}$ NSs only have the $\mathrm{Co}^{2+} \mathrm{O}_{\mathrm{h}}$ sites, suggesting the amorphization treatment could partially change the symmetry of the ligand field around Co atoms.

Electrochemical performance of a-CoO NSs toward Li-S batteries. To understand the effects of a-CoO NSs and c-CoO NSs hosts on the Li-S chemistry, electrochemical measurements were systematically carried out to compare the performance of Sloaded rGO/a-CoO NSs (S@rGO/a-CoO NSs), rGO/c-CoO NSs (S@rGO/c-CoO NSs), and S@rGO electrodes. The sulfur contents are estimated by thermogravimetric analysis (TGA, Supplementary Fig. 6). In typical CV (Fig. 3a) profiles, the positive shift in the reduction peaks and a negative shift in the oxidation peaks of the $\mathrm{rGO} / \mathrm{a}-\mathrm{CoO}$ NSs electrode indicate the improved redox kinetics compared with the $\mathrm{rGO} / \mathrm{c}-\mathrm{CoO} \mathrm{NS}$ electrode. It is noteworthy that the $\mathrm{rGO} / \mathrm{a}-\mathrm{CoO}$ NSs electrode shows obviously higher peak current intensity at $2.04 \mathrm{~V}$ than the $\mathrm{rGO} / \mathrm{c}-\mathrm{CoO} \mathrm{NSs}$ electrode, which indicates that the conversion rate of soluble LiPSs to insoluble $\mathrm{Li}_{2} \mathrm{~S}_{2} / \mathrm{Li}_{2} \mathrm{~S}$ was particularly improved ${ }^{39}$. The improved reduction rate of LiPSs has been demonstrated to be a crucial step in reducing the amount of LiPSs diffused in the electrolyte and improving the utilization of $S$ and cycling life of Li-S batteries ${ }^{12}$. Galvanostatic charge/discharge measurements (Fig. 3b) were further performed to confirm the above results, in which the initial voltage-capacity curves of three electrodes exhibit typically two discharge and one charge plateaus, corresponding to the charge and discharge processes respectively, matching well with the $\mathrm{CV}$ results. A much smaller overpotential between discharge and charge plateau at the region of $2.0-2.2 \mathrm{~V}$ of S@rGO/a-CoO NSs electrode suggests a kinetically favorable redox reaction of Li-S chemistry. Moreover, the charge/discharge plateau can be well maintained during cycling (Supplementary Fig. 7), reflecting the excellent capability toward LiPSs adsorption/conversion to suppress the shuttle effect. In addition, the EIS of three electrodes (Supplementary Fig. 8) has ascertained the favorable redox reactions, in which S@rGO/a-CoO NSs display the lowest charge-transfer resistance compared to the other two electrodes.

To further study their electrochemical performance at high current densities, the rate properties of the three electrodes (Fig. 3c) are measured with the rates varying from 0.2 to $10.0 \mathrm{C}$. The $\mathrm{rGO} / \mathrm{a}-\mathrm{CoO}$ NSs electrode achieves highly reversible discharge capacities of $1526.3,1222.6,1039.5,938.3$, and $702.3 \mathrm{mAh} \mathrm{g}^{-1}$ at rates of $0.2,0.5,1,2$, and $5 \mathrm{C}$, respectively. Even at high rates of 8 and $10 \mathrm{C}$, the $\mathrm{rGO} / \mathrm{a}-\mathrm{CoO}$ NSs electrode still remains an impressive capacity of 451.5 and $306.6 \mathrm{mAh} \mathrm{g}^{-1}$, respectively, representing a superior rate performance among the ever-reported metal oxide-based compounds for Li-S batteries (Fig. 3d, Supplementary Table 4$)^{7-9,19,40-45}$. Moreover, at the high rate, it still possesses an obvious charge/discharge voltage plateaus (Supplementary Fig. 9), meaning the electrochemical redox kinetics have been substantially boosted with the addition of a-CoO NSs. In addition, the specific capacities of $\mathrm{rGo}$, a-CoO, and c-Co are also studied, which suggests their contributions to the whole capacity are negligible (Supplementary Fig. 10). Figure $4 \mathrm{e}$ shows the long-term cyclability of $\mathrm{rGO} / \mathrm{a}-\mathrm{CoO}$ NSs electrode at $1 \mathrm{C}$, with an initial capacity as high as $1248.2 \mathrm{~mA} \mathrm{~h} \mathrm{~g}^{-1}$ after the initial activation cycle, and retains $83.1 \%$ of the initial capacity $\left(1037.3 \mathrm{mAh} \mathrm{g}^{-1}\right)$ after 500 cycles. Moreover, the discharge-charge voltage curve of the S@rGO/a-CoO NSs electrode can be well maintained after 500 cycles (Supplementary Fig. 11), further demonstrating its robustness for the chemical adsorption and conversion during cycling.

Since the electrochemical performance of Li-S battery is typically related to the adsorption properties of electrodes ${ }^{14,46}$, we 


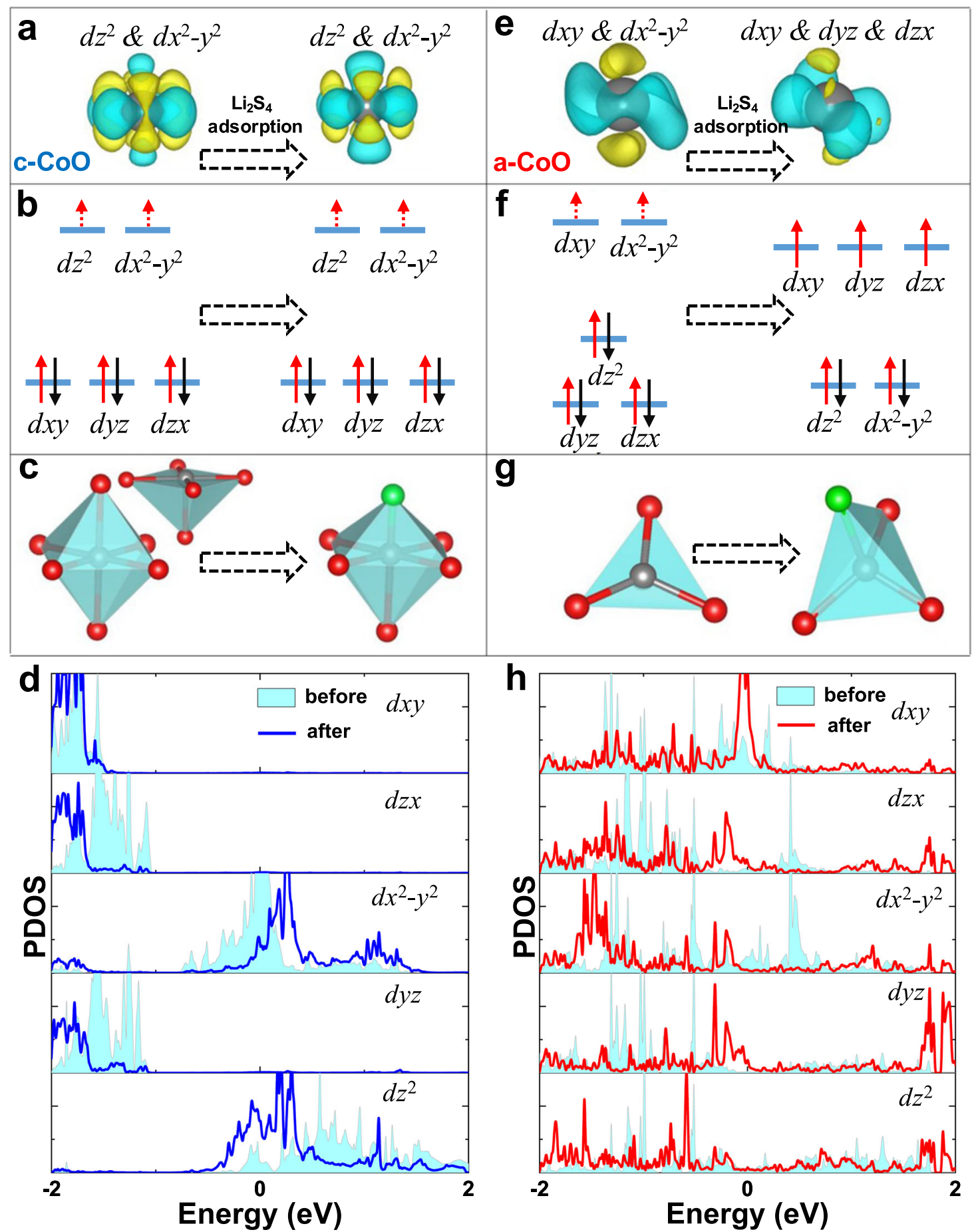

Fig. 4 Theoretical calculations of the reaction mechanism. The calculated deformation change density for $\mathrm{Co}$ of $\mathbf{a} \mathrm{c}-\mathrm{CoO}$ and $\mathbf{e}$ a-CoO before (left) and after (right) $\mathrm{Li}_{2} \mathrm{~S}_{4}$ adsorption; the schematic of $d$ orbitals for $\mathrm{Co}$ of $\mathbf{b} \mathrm{c}-\mathrm{CoO}$ and $\mathbf{f}$ a-CoO before (left) and after(right) $\mathrm{Li}_{2} \mathrm{~S}_{4}$ adsorption; the coordination structure for $\mathrm{Co}$ of $\mathbf{c} \mathrm{c}-\mathrm{CoO}$ and $\mathbf{g}$ a-CoO before (left) and after (right) $\mathrm{Li}_{2} \mathrm{~S}_{4}$ adsorption; the PDOS for Co of $\mathbf{d} \mathrm{c}-\mathrm{CoO}_{0}$ before and after $\mathrm{Li}_{2} \mathrm{~S}_{4}$ adsorption respectively and $\mathbf{h}$ a-CoO before and after $\mathrm{Li}_{2} \mathrm{~S}_{4}$ adsorption respectively. Red balls: O; grey balls, Co; green balls, $\mathrm{S}$; yellow areas: charge accumulation; navy area: charge depletion; Fermi level in this work set to zero; the short arrow in Fig. 4b, f represents the semi-occupied state of the electron.

further compare their adsorption capabilities toward LiPSs. Therefore, HADDF-STEM was employed to map the distribution of S element in both S@rGO/a-CoO NSs and S@rGO/c-CoO NSs electrodes in the middle of discharge (Supplementary Figs. 12 and 13), which shows that the average atomic ratio of S:Co element in S@rGO/a-CoO NSs electrode is higher than that in S@rGO/c$\mathrm{CoO}$ NSs electrode. Moreover, adsorption tests toward $\mathrm{Li}_{2} \mathrm{~S}_{6}$ species were conducted with the same total surface area of a-CoO
NSs, c-CoO NSs, and rGO (Supplementary Fig. 14). Apparently, the solution with a-CoO NSs becomes much clearer without obvious color, suggesting the adsorption capability of CoO NSs is improved after amorphization.

Theoretical calculations. To understand the mechanism of the improved electrochemical performance of the a-CoO NSs, DFT 
calculations were employed to investigate the properties of the c$\mathrm{CoO}$ and a-CoO surfaces respectively, as well as the interaction between their surfaces and sulfide species $\left(\operatorname{Li}_{2} \mathrm{~S}_{x}, x=1,2,4,8\right)$. The models of the a-CoO and $\mathrm{c}-\mathrm{CoO}$ surfaces are displayed in Supplementary Fig. $15 \mathrm{a}, \mathrm{b}$, where the a-CoO possesses partial distorted or truncated $\mathrm{CoO}_{4}$ tetrahedrons, matching well with the simulated results of experimental Co L-edge XANES data in Fig. 2f. The changed symmetry of the ligand field around Co atoms would influence their properties of $d$ electrons. To describe the strong-correlation effects among $d$ electrons of Co, the DFT $+\mathrm{U}$ method with $U_{\text {eff }}=3.3 \mathrm{eV}$ for Co was further employed. The deformation charge density of $\mathrm{c}-\mathrm{CoO}$ and a-CoO (Supplementary Fig. $15 \mathrm{c}, \mathrm{d})$ show that the electron loss of $\mathrm{Co}$ atoms in a-CoO $\left(\mathrm{Co}_{\mathrm{AM}}\right)$ is much different from the ones in $\mathrm{c}-\mathrm{CoO}\left(\mathrm{Co}_{\mathrm{CR}}\right)$. Specifically, the electrons in high-energy orbitals are easy to loss, therefore, the $\mathrm{Co}_{\mathrm{CR}}$ should lose electrons on $d z^{2}$ and $d x^{2}-y^{2}$ orbitals, which are the highest occupied orbitals in the Co-O octahedron structure (or truncated octahedron) as in left of Fig. $4 \mathrm{a}, \mathrm{b}$. However, the electrons of $\mathrm{Co}_{\mathrm{AM}}$ should be lost from the high energy $d x y$ and $d x^{2}-y^{2}$ orbitals owing to the re-distributed of $d$ orbitals as in left of Fig. $4 \mathrm{e}-\mathrm{g}$. Projected density of state (PDOS) of $\mathrm{Co}$ in Fig. $4 \mathrm{~d}$, h, shows the orbital distributions and well consistent with the schematic in Fig. 4b, f, respectively. It is worth mentioning that such a difference in orbital distribution can influence the behavior of LiPSs adsorption.

To this end, we calculated the binding energy of $\mathrm{Li}_{2} \mathrm{~S}_{x}$ on c$\mathrm{CoO}(100)$ and a-CoO surfaces, respectively, as in Supplementary Fig. 16. Obviously, the $\mathrm{Li}_{2} \mathrm{~S}_{x}$ on a-CoO surfaces have been distorted, and $\mathrm{Li}_{2} \mathrm{~S}_{x}$ on $\mathrm{c}-\mathrm{CoO}$ can maintain their geometric structures. Such difference can be reasonably explained by the strong adsorption ability of a-CoO toward $\mathrm{Li}_{2} \mathrm{~S}_{x}$, as shown in Supplementary Table 5. The binding energies of $\mathrm{Li}_{2} \mathrm{~S}_{x}$ on a-CoO surfaces are much higher than those on c-CoO (Supplementary Fig. 17), which is consistent to the results of the adsorption tests in Supplementary Figs. 12-14. To explore the origin of the enhanced binding energy by a-CoO, we investigated the electrons transfer between $\mathrm{Li}_{2} \mathrm{~S}_{4}$ and a-CoO as well as c-CoO, respectively in Supplementary Fig. 18a, b. More electrons were transferred from a-CoO to $\mathrm{Li}_{2} \mathrm{~S}_{4}$, which can strengthen the Coulomb interactions between negative charged $S$ and positive charged $\mathrm{Co}$ atoms, as well as $\mathrm{O}$ and $\mathrm{Li}$. The deformation charge density for Co atoms in $\mathrm{Li}_{2} \mathrm{~S}_{4}$ adsorbed sites was further calculated to deeply understand the electron transfer. In the right of Fig. $4 \mathrm{a}$, e, the electron loss of $\mathrm{Co}_{\mathrm{CR}}$ after $\mathrm{Li}_{2} \mathrm{~S}_{4}$ adsorption is almost the same as ones without $\mathrm{Li}_{2} \mathrm{~S}_{4}$, which can be attributed to the hardly changed orbital distributions. The PDOS in Fig. 4 d indicates that only few $t_{2 \mathrm{~g}}(d x y, d x z, d y z)$ electrons of $\mathrm{Co}_{\mathrm{CR}}$ lost after $\mathrm{Li}_{2} \mathrm{~S}_{4}$ adsorption because $t_{2 \mathrm{~g}}$ electrons located at a lower energy level (under Fermi level) are stable. In contrast, owing to the re-distribution of $d$ orbitals for a-CoO, the $d$ orbitals of active Co split to $t_{2 \mathrm{~g}}(d x y, d x z$, $d y z)$ and $e_{\mathrm{g}}\left(d z^{2}, d x^{2}-y^{2}\right)$ after $\mathrm{Li}_{2} \mathrm{~S}_{4}$ adsorption (Fig. 4f). The $t_{2 \mathrm{~g}}$ electrons have high energy, which can be lost easier than $e_{\mathrm{g}}$. One should be noticed that there are three electrons on the higher energy level of $\mathrm{Co}_{\mathrm{AM}}$, and only one electron on the higher level of $\mathrm{Co}_{\mathrm{CR}}$. Therefore, the $\mathrm{Co}_{\mathrm{AM}}$ can lose more electrons to react with $\mathrm{Li}_{2} \mathrm{~S}_{4}$. Moreover, the PDOS in Fig. $4 \mathrm{~h}$ also demonstrated that the Co $d$ orbitals of a-CoO samples changed after $\mathrm{S}$ adsorption, and such change is consistent with the schematic in Fig. 4f. The PDOS in Supplementary Fig. $18 \mathrm{c}$ and d provide compelling evidence, that is, the more $d$ orbitals of $\mathrm{Co}_{\mathrm{AM}}$ are overlapped with $p$ orbital of $S$, which generated higher binding energy.

\section{Discussion}

In summary, we demonstrate the strategy of amorphizationinduced surface electronic states modulation in $\mathrm{CoO}$ nanosheets, which can significantly improve the performance of Li-S batteries. X-ray absorption spectroscopy analysis reveals that the coordination structures of $\mathrm{CoO}$ nanosheets have been changed after amorphization. DFT studies further indicate that amorphization-induced redistribution of $d$ orbitals can strengthen the binding energy of LiPSs. The a-CoO NSs delivers a high-rate performance with $1100 \mathrm{mAh} \mathrm{g}^{-1}$ at $1 \mathrm{C}$ and retains $700 \mathrm{mAh} \mathrm{g}^{-1}$ even at $5 \mathrm{C}$. More importantly, the a-CoO NSs applied in $\mathrm{Li}-\mathrm{S}$ batteries have an ultralong cycle life (500 cycles) with a decay rate of only $0.034 \%$ per cycle. This work provides a novel guideline for rational modulation of surface electronic states to optimize the adsorption of LiPSs for high energy density Li-S batteries.

\section{Methods}

Chemicals. Cobalt(II) acetylacetonate $\left(\mathrm{Co}(\mathrm{acac})_{2}\right)$ were purchased from Alfa Aesar. Ethanol and $\mathrm{KBr}$ were obtained from Shanghai Chemical Reagents, China Anhydrous ethanol and ammonia solution $\left(\mathrm{NH}_{4} \mathrm{OH}\right)$ were purchased from Sinopharm Chemical Reagent Co., Ltd. (Shanghai, China). Deionized (DI) water from Milli-Q System (Millipore, Billerica, MA) was used in all our experiments.

Synthesis of a-CoO NSs. In a typical procedure, $10 \mathrm{mg} \mathrm{Co(acac})_{2}$ was dissolved in $9 \mathrm{~mL}$ ethanol. After stirring to form a homogeneous solution, $\mathrm{KBr}$ aqueous solution $\left(12 \mathrm{mg} \mathrm{mL}^{-1}, 1 \mathrm{~mL}\right)$ was injected into the mixed solution slowly under ultrasound at room temperature. Next, the mix solution was under vigorous stirring for $2 \mathrm{~h}$ at $35^{\circ} \mathrm{C}$. Then the sample was dried in an oven at $80^{\circ} \mathrm{C}$ for $3 \mathrm{~h}$. The sample was placed in a tube furnace and heated to $240^{\circ} \mathrm{C}$ (heating rate $5^{\circ} \mathrm{C} / \mathrm{min}$ ) in air for 80 min. After washing with ethanol and DI water several times, the a-CoO NSs were finally obtained and dissolved in ethanol for further characterization.

Synthesis of c-CoO NSs. In a typical procedure, the as-obtained a-CoO NSs were dried in a vacuum at $-35^{\circ} \mathrm{C}$ for overnight. Then the powder of a-CoO NSs (100 $\mathrm{mg}$ ) was placed in a tube furnace and heated to $400^{\circ} \mathrm{C}$ (heating rate $15^{\circ} \mathrm{C} / \mathrm{min}$ ) for $30 \mathrm{~min}$ in a stream of Ar to yield c-CoO NSs.

Synthesis of reduced graphene oxide (rGO). Graphene oxide (GO) was prepared by Hummer's method ${ }^{47}$. The rGO was synthesized using a hydrothermal method. Typically, $25 \mathrm{~mL}$ anhydrous ethanol was mixed with $14 \mathrm{~mL}$ of $5 \mathrm{mg} \mathrm{mL}^{-1} \mathrm{GO}$ solution in a flask, followed by the addition of $1.2 \mathrm{~mL} \mathrm{NH}_{4} \mathrm{OH}$. The mixed solution was vigorously stirred for $10 \mathrm{~h}$ at $80^{\circ} \mathrm{C}$, before transferring to a $40 \mathrm{~mL}$ autoclave. The hydrothermal reaction was performed at $150^{\circ} \mathrm{C}$ for $3 \mathrm{~h}$.

Characterization. Transmission electron microscopy (TEM) images of samples were recorded by Hitachi $\mathrm{H}-7650$ at an acceleration voltage of $100 \mathrm{kV}$. Aberrationcorrected HAADF-STEM images were recorded with a JEOL JEM-2010 $\mathrm{LaB}_{6}$ highresolution transmission electron microscope, which operated at $200 \mathrm{kV}$. Before HADDF-STEM tests, the S@rGO/a-CoO NSs and S@rGO/c-CoO NSs electrodes were washed once with tetrahydrofuran and then ultrasonically dispersed. Atomic force microscopy (AFM) image was captured by Dimension ICON with Bruker NanoScope V controller with Scan Asyst mode. Powder XRD patterns of all samples were tested on a Rigaku Miniflex-600 operated at $40 \mathrm{kV}$ voltage and $15 \mathrm{~mA}$ current with $\mathrm{CuKa}$ radiation $(\lambda=0.15406 \mathrm{~nm})$. X-ray photoelectron spectroscopy (XPS) was collected on scanning X-ray microprobe (PHI 5000 Verasa, ULAC-PHI, Inc.) by $\mathrm{Al} \mathrm{Ka}$ radiation and the $\mathrm{C} 1 \mathrm{~s}$ peak located at $284.8 \mathrm{eV}$ as standard. XAFS measurements and data analysis: XAFS spectra at the Co K-edge were recorded at BL14W1 station in Shanghai Synchrotron Radiation Facility (SSRF). The electron storage ring of SSRF was performed at $3.5 \mathrm{GeV}$ with a maximum of current of 250 $\mathrm{mA}$. The Co K-edge XANES data were recorded in a fluorescence mode and Co foil, $\mathrm{CoO}$, and $\mathrm{Co}_{3} \mathrm{O}_{4}$ were used as references. The Raman spectrum was performed on LabRamHR. The obtained $\mathrm{N}_{2}$ adsorption-desorption isotherms were evaluated to give the Brunauer-Emmett-Teller (BET) specific surface area. Thermogravimetric analysis (TGA, SDT-Q600) was performed under nitrogen atmosphere by heating from RT to $500{ }^{\circ} \mathrm{C}$ at $10^{\circ} \mathrm{C} \mathrm{min}{ }^{-1}$ to measure the sulfur content in the cathode materials with the samples degassed at $30^{\circ} \mathrm{C}$ for $3 \mathrm{~h}$.

L-edge XANES measurement and simulation. The Co L-edge XANES spectra were measured in the Hefei NSRL station. The experimental condition was at room temperature. The simulations of Co L-edge XANES spectra were using CTM4XAS program and the adjusted parameters are provided in Supplementary Tables 2 and 3 , where the temperature for the simulations is $300 \mathrm{~K}^{48-50}$.

Electrochemical measurements. Sulfur was loaded into rGO, rGO/a-CoO NSs and $\mathrm{rGO} / \mathrm{c}-\mathrm{CoO}$ NSs by a melt-diffusion method in a glass tube with controlled weight ratios (rGO: a-CoO NSs/c-CoO NSs $=4: 1$ ). The melt-diffusion process was conducted at $155^{\circ} \mathrm{C}$ for $10 \mathrm{~h}$. To fabricate the working electrodes, a slurry was prepared by mixing 80 wt\% active materials (S@rGO, S@rGO/a-CoO NSs, S@rGO/ 
c-CoO NSs), $10 \mathrm{wt} \%$ super $\mathrm{P}$, and $10 \mathrm{wt} \%$ polyvinylidene fluoride, and coating them on a carbon-coated aluminum foil. The prepared electrodes were dried in a vacuum oven at $60^{\circ} \mathrm{C}$ for $24 \mathrm{~h}$. The electrochemical performances were finally measured with using coin-type half cells (CR 2016). The coin-type half cells were assembled in a glovebox with lithium metal foil as the anode and $1 \mathrm{~mol} \mathrm{~L}^{-1} \mathrm{LiTFSI}$ and DME: DOL $(v / v=1: 1)$ that containing $1 \mathrm{wt} \% \mathrm{LiNO}_{3}$ as the electrolyte. The electrolyte dosage used in the half cells was $\sim 10 \mu \mathrm{L} \mathrm{mg}^{-1}$ of electrolyte/sulfur (E/S) ratio. The coin-cell was tested with galvanostatic cycling on a battery test system (LAND CT2001) with a voltage window of $1.7-2.8 \mathrm{~V}\left(\mathrm{vs} . \mathrm{Li}^{+} / \mathrm{Li}\right)$ in lithium-ion battery at room temperature. Cyclic voltammetry (CV, CHI 660e) was carried out at a scan rate of $0.1 \mathrm{mV} \mathrm{s}^{-1}$ from 1.7 to $2.8 \mathrm{~V}$ (vs. $\mathrm{Li}^{+} / \mathrm{Li}$ ) at room temperature. Electrochemical impedance spectroscopy (EIS) tests were conducted on a Solartron 1470 E electrochemical workstation, using potentiostatic mode at open circuit potential. The sinusoidal voltage with $10 \mathrm{mV}$ amplitude and the scanning frequency from $1 \mathrm{~Hz}$ to $100 \mathrm{kHz}$ was used.

Visualized adsorption experiment. $\mathrm{Li}_{2} \mathrm{~S}_{6}$ solution was prepared by adding a mixture of lithium sulfide and sulfur powders with a molar ratio of 1:5 into tetraglyme, followed by vigorous magnetic stirring for $24.0 \mathrm{~h}$. The concentration of $\mathrm{Li}_{2} \mathrm{~S}_{6}$ solution was set as $0.5 \mathrm{mmol} \mathrm{L}^{-1}$. The rGOs, a-CoO NSs, and c-CoO NSs with the same total surface area (set as $0.5 \mathrm{~m}^{2}$, equal to that calculated from the product of the surface area and sample weight) were added into $1.0 \mathrm{~mL} \mathrm{Li}_{2} \mathrm{~S}_{6}$ solution, respectively. These mixtures were vigorously stirred to ensure thorough adsorption and were then kept statically until all the suspended particles settled down.

Theoretical method. First principle calculations were performed by density function theory (DFT) code of Vienna ab initio Simulation Package (VASP) ${ }^{51}$. The generalized gradient approximation (GGA) with the function of

Perdew-Burke-Ernzerhof ${ }^{52}$ was used to describe the electron exchange corrections. Some parameters were set as follow: cutoff energy, $550 \mathrm{eV} ; k$-mesh, $5 \times 5 \times 1 ; 20 \AA$ vacuum space for all surfaces; convergence criteria, $-0.01 \mathrm{eV} / \AA$ and $10^{-5} \mathrm{eV}$ for force and energy, respectively. The weak interactions between $\mathrm{CoO}$ and polysulfides have been considered through the DFT-D3 scheme ${ }^{53}$. To describe strongcorrelation effects among $d$ electrons of Co, the Hubbard model has been employed $(\mathrm{DFT}+\mathrm{U})^{54}$, and the $U_{\text {eff }}$ for Co is $3.3 \mathrm{eV}^{55}$. The amorphous models were relaxed by ab initio molecular dynamic (AIMD) simulations ${ }^{56}$ under $800 \mathrm{~K}$ and a time step of $1 \mathrm{fs}$ lasting $5 \mathrm{ps}$. VASPKIT, an interface for VASP calculation, provides great convenience in data processing and graphic production ${ }^{57}$

The binding energy for polysulfide (LiPS) is calculated as Eq. (1):

$$
E_{b}=E_{L i P S @ C o O}-E_{C o O}-E_{L i P S}
$$

where $E_{\mathrm{LiPS} @ \mathrm{CoO}}, E_{\mathrm{CoO}}, E_{\mathrm{LiPS}}$ represent the total energies of LiPS+ adsorbed samples, $\mathrm{CoO}$ surfaces (c-CoO or a-CoO), and LiPS, respectively. On the basis of this definition, a more negative value of $E_{\mathrm{b}}$ represents a stronger binding ability as well as greater thermodynamic stability.

The deformation charge density $\left(\rho_{\mathrm{d}}\right)$ can describe the charge difference (or charge transfer) between atoms and compounds, and calculated as Eq. (2): $\rho_{\mathrm{d}}=\rho_{\text {scf }}-\rho_{\text {atom }}$, where $\rho_{\text {scf }}$ and $\rho_{\text {atom }}$ are the charge density of the whole system and the total charge densities of each atom in the whole system, respectively. The charge density difference $\left(\rho_{c}\right)$, described the charge redistribution caused by the adsorption of LiPSs, was calculated by Eq. (3): $\rho_{\mathrm{c}}=\rho_{\mathrm{LiPSs} @ \mathrm{CoO}}-\rho_{\mathrm{CoO}}-\rho_{\mathrm{LiPS}}$, where $\rho_{\mathrm{LiPS} @ \mathrm{CoO}}, \rho_{\mathrm{CoO}}, \rho_{\mathrm{LiPSs}}$ represent the total charge density of of LiPSs adsorbed samples, $\mathrm{CoO}$ surfaces (c-CoO or a-CoO) and LiPSs, respectively.

Details of amorphous models have been constructed by the following step: (1) create a pristine c-CoO (100) surface, (2) randomly delete some Co and O atoms, (3) relax these models by using AIMD simulations under $800 \mathrm{~K}$, (4) optimize the relaxed structures from AIMD by using DFT calculations, (5) select the best one among these structures, and (6) calculate the electronic properties of selected models and the adsorption of polysulfides.

\section{Data availability}

The data that support the findings of this study are available from the corresponding authors upon reasonable request.

\section{Code availability}

The code used in this study is free availability in the website https://www.euspec.eu/codedatabase.

Received: 16 September 2020; Accepted: 16 April 2021; Published online: 25 May 2021

\section{References}

1. Manthiram, A., Chung, S. H. \& Zu, C. Lithium-sulfur batteries: progress and prospects. Adv. Mater. 27, 1980-2006 (2015).
2. Seh, Z. W., Sun, Y., Zhang, Q. \& Cui, Y. Designing high-energy lithium-sulfur batteries. Chem. Soc. Rev. 45, 5605-5634 (2016).

3. Peng, H.-J., Huang, J.-Q., Cheng, X.-B. \& Zhang, Q. Review on high-loading and high-energy lithium-sulfur batteries. Adv. Energy Mater. 7, 1700260 (2017).

4. Cao, K. et al. Encapsulating sulfur in $\delta-\mathrm{MnO} 2$ at room temperature for $\mathrm{Li}-\mathrm{S}$ battery cathode. Energy Storage Mater. 9, 78-84 (2017).

5. Zheng, J. et al. Lewis acid-base interactions between polysulfides and metal organic framework in lithium sulfur batteries. Nano Lett. 14, 2345-2352 (2014).

6. Liang, X. et al. Tuning transition metal oxide-sulfur interactions for long life lithium sulfur batteries: the "Goldilocks" principle. Adv. Energy Mater. 6, 1501636 (2016).

7. Wei Seh, Z. et al. Sulphur-TiO2 yolk-shell nanoarchitecture with internal void space for long-cycle lithium-sulphur batteries. Nat. Commun. 4, 1331 (2013).

8. Chang, Z. et al. Co3O4 nanoneedle arrays as a multifunctional "superreservoir” electrode for long cycle life Li-S batteries. J. Mater. Chem. A 5, 250-257 (2017).

9. Liang, X. et al. A highly efficient polysulfide mediator for lithium-sulfur batteries. Nat. Commun. 6, 5682 (2015).

10. Zhang, Q. et al. Understanding the anchoring effect of two-dimensional layered materials for lithium-sulfur batteries. Nano Lett. 15, 3780-3786 (2015).

11. Pang, Q., Liang, X., Kwok, C. Y. \& Nazar, L. F. Advances in lithium-sulfur batteries based on multifunctional cathodes and electrolytes. Nat. Energy 1, 16132 (2016)

12. Shen, S. et al. Implanting niobium carbide into trichoderma spore carbon: a new advanced host for sulfur cathodes. Adv. Mater. 31, e1900009 (2019).

13. Zhong, Y. et al. Surface chemistry in cobalt phosphide-stabilized lithiumsulfur batteries. J. Am. Chem. Soc. 140, 1455-1459 (2018).

14. Zhou, J. et al. Deciphering the modulation essence of $\mathrm{p}$ bands in Co-based compounds on Li-S chemistry. Joule 2, 2681-2693 (2018).

15. Patil, S. B. et al. Exfoliated 2D lepidocrocite titanium oxide nanosheets for high sulfur content cathodes with highly stable Li-S battery performance. ACS Energy Lett. 3, 412-419 (2018).

16. Lin, $\mathrm{H}$. et al. Elucidating the catalytic activity of oxygen deficiency in the polysulfide conversion reactions of lithium-sulfur batteries. Adv. Energy Mater. 8, 1801868 (2018).

17. Bao, W. et al. Facile synthesis of crumpled nitrogen-doped MXene nanosheets as a new sulfur host for lithium-sulfur batteries. Adv. Energy Mater. 8 1702485 (2018).

18. $\mathrm{Xu}, \mathrm{L}$. et al. Oxygen vacancies on layered niobic acid that weaken the catalytic conversion of polysulfides in lithium-sulfur batteries. Angew. Chem. Int. Ed. 58, 11491-11496 (2019).

19. Yang, W. et al. Tin intercalated ultrathin MoO3 nanoribbons for advanced lithium-sulfur batteries. Adv. Energy Mater. 9, 1803137 (2019)

20. Sun, W. et al. An amorphous lanthanum-iridium solid solution with an open structure for efficient water splitting. J. Mater. Chem. A 8, 12518-12525 (2020).

21. Zhang, $X$. et al. Lithiation-induced amorphization of Pd3P2S8 for highly efficient hydrogen evolution. Nat. Catal. 1, 460-468 (2018).

22. Gao, J. et al. Breaking long-range order in iridium oxide by alkali ion for efficient water oxidation. J. Am. Chem. Soc. 141, 3014-3023 (2019).

23. $\mathrm{Wu}, \mathrm{G}$. et al. A general synthesis approach for amorphous noble metal nanosheets. Nat. Commun. 10, 4855 (2019).

24. Sun, R. et al. Amorphous metal oxide nanosheets featuring reversible structure transformations as sodium-ion battery anodes. Cell Rep. Phys. Sci. 1, 100118 (2020).

25. Ravindra, A. V., Behera, B. C. \& Padhan, P. Laser induced structural phase transformation of cobalt oxides nanostructures. J. Nanosci. Nanotechnol. 14, 5591-5595 (2014).

26. Lin, Z., Du, C., Yan, B. \& Yang, G. Two-dimensional amorphous CoO photocatalyst for efficient overall water splitting with high stability. J. Catal. 372, 299-310 (2019).

27. Wang, S. et al. Nanoparticle cookies derived from metal-organic frameworks controlled synthesis and application in anode materials for lithium-ion batteries. Small 12, 2365-2375 (2016).

28. Kaneti, Y. V. et al. Fabrication of an MOF-derived heteroatom-doped Co/ $\mathrm{CoO} /$ carbon hybrid with superior sodium storage performance for sodiumion batteries. J. Mater. Chem. A 5, 15356-15366 (2017).

29. Chen, Z. et al. Amorphous cobalt oxide nanoparticles as active wateroxidation catalysts. Chem CatChem 9, 3641-3645 (2017).

30. Risch, M. et al. Structural changes of cobalt-based perovskites upon water oxidation investigated by EXAFS. J. Phys. Chem. C 117, 8628-8635 (2013).

31. Kanan, M. W. et al. Structure and valency of a cobalt-phosphate water oxidation catalyst determined by in situ X-ray spectroscopy. J. Am. Chem. Soc. 132, 13692-13701 (2010). 
32. Risch, M. et al. Water oxidation by electrodeposited cobalt oxides-role of anions and redox-inert cations in structure and function of the amorphous catalyst. ChemSusChem 5, 542-549 (2012).

33. $\mathrm{Hu}, \mathrm{F}$. et al. Amorphous metallic NiFeP: a conductive bulk material achieving high activity for oxygen evolution reaction in both alkaline and acidic media. Adv. Mater. 29, 1606570 (2017).

34. Li, B. et al. Amorphous nickel-iron oxides/carbon nanohybrids for an efficient and durable oxygen evolution reaction. Nano Res. 10, 3629-3637 (2017).

35. $\mathrm{Xu}$, J. et al. Visible-light-driven overall water splitting boosted by tetrahedrally coordinated blende cobalt(II) oxide atomic layers. Angew. Chem. Int. Ed. 58, 3032-3036 (2019).

36. Ghiasi, M. et al. Mn and Co charge and spin evolutions in LaMn1-xCoxO3 nanoparticles. J. Phys. Chem. C 120, 8167-8174 (2016).

37. $\mathrm{Mu}, \mathrm{C}$. et al. Rational design of spinel cobalt vanadate oxide $\mathrm{Co} 2 \mathrm{VO} 4$ for superior electrocatalysis. Adv. Mater. 32, e1907168 (2020).

38. Wang, Y. et al. Bio-inspired leaf-mimicking nanosheet/nanotube heterostructure as a highly efficient oxygen evolution catalyst. Adv. Sci. 2, 1500003 (2015).

39. Zhong, Y. et al. Confining sulfur in integrated composite scaffold with highly porous carbon fibers/vanadium nitride arrays for high-performance lithiumsulfur batteries. Adv. Funct. Mater. 28, 1706391 (2018).

40. Li, Z., Zhang, J. \& Lou, X. W. Hollow carbon nanofibers filled with $\mathrm{MnO} 2$ nanosheets as efficient sulfur hosts for lithium-sulfur batteries. Angew. Chem. Int. Ed. 54, 12886-12890 (2015).

41. $\mathrm{Wu}, \mathrm{S}$. et al. Porous hollow carbon nanospheres embedded with welldispersed cobalt monoxide nanocrystals as effective polysulfide reservoirs for high-rate and long-cycle lithium-sulfur batteries. J. Mater. Chem. A 5, 17352-17359 (2017).

42. Lu, K. et al. Manipulating polysulfide conversion with strongly coupled $\mathrm{Fe}_{3} \mathrm{O}_{4}$ and nitrogen doped carbon for stable and high capacity lithium-sulfur batteries. Adv. Funct. Mater. 29, 1807309 (2019).

43. Jiao, L. et al. Capture and catalytic conversion of polysulfides by in situ built TiO2-MXene heterostructures for lithium-sulfur batteries. Adv. Energy Mater. 9, 1900219 (2019).

44. Wang, Y. et al. Enhancing catalytic activity of titanium oxide in lithium-sulfur batteries by band engineering. Adv. Energy Mater. https://doi.org/10.1002/ aenm.201900953 (2019).

45. Wang, S. et al. Designing a highly efficient polysulfide conversion catalyst with paramontroseite for high-performance and long-life lithium-sulfur batteries. Nano Energy 57, 230-240 (2019).

46. Zhang, L. et al. High-content of sulfur uniformly embedded in mesoporous carbon: a new electrodeposition synthesis and an outstanding lithium-sulfur battery cathode. J. Mater. Chem. A 5, 5905-5911 (2017).

47. Hummers, W. S. \& Offeman, R. E. Preparation of graphitic oxide. J. Am. Chem. Soc. 80, 1339-1339 (1958).

48. Stavistki, E. \& de Groot, F. M. F. The CTM4XAS program for EELS and XAS spectral shape analysis of transition metal L edges. Micron 41, 687-694 (2010).

49. Csiszar, S. I. et al. Controlling orbital moment and spin orientation in $\mathrm{CoO}$ layers by strain. Phys. Rev. Lett. 95, 187205 (2005).

50. Groot, F. D. Multiplet effects in X-ray spectroscopy. Coord. Chem. Rev. 249, 31-63 (2005)

51. Kresse, G. \& Furthmüller, J. Efficient iterative schemes for ab initio totalenergy calculations using a plane-wave basis set. Phys. Rev. B 54, 11169-11186 (1996).

52. Perdew, J. P., Burke, K. \& Ernzerhof, M. Generalized gradient approximation made simple. Phys. Rev. Lett. 77, 3865-3868 (1996).

53. Grimme, S., Antony, J., Ehrlich, S. \& Krieg, H. A consistent and accurate ab initio parametrization of density functional dispersion correction (DFT-D) for the 94 elements H-Pu. J. Chem. Phys. 132, 154104 (2010).
54. Dudarev, S. L. et al. Electron-energy-loss spectra and the structural stability of nickel oxide: an LSDA+U study. Phys. Rev. B 57, 1505 (1998).

55. Wang, L., Maxisch, T. \& Ceder, G. Oxidation energies of transition metal oxides within the GGA+U framework. Phys. Rev. B 73, 195107 (2006).

56. Nosé, S. A unified formulation of the constant temperature molecular dynamics methods. J. Chem. Phys. 81, 511-519 (1984).

57. Wang, V. et al. VASPKIT: a user-friendly interface facilitating highthroughput computing and analysis using VASP code. Preprint at https:// arxiv.org/abs/1908.08269 (2019).

\section{Acknowledgements}

This work was supported by the National Key R\&D Program of China (2017YFA0700104, 2018YFA0702001, and 2017YFA0206703), National Natural Science Foundation of China (21871238, 11704365, and 21975244), Youth Innovation Promotion Association of the Chinese Academy of Science (2018494), and Fundamental Research Funds for the Central Universities (WK2060000016).

\section{Author contributions}

X.H. and G.M.W. conceived the idea and co-wrote the paper; R.L. performed experi mental work with the help of J.Z.; D.R. performed the computational work with the discussion with G.M.W.; H.L. carried out the AFM characterization; C.W., W.Y., X.Z., and P.C. performed the XAS simulations; G.W., Z.Z., X.H., and R.S. helped with the modification of the paper. G.Z.W. and Y.W. discussed the results and commented on the paper.

\section{Competing interests}

The authors declare no competing interests.

\section{Additional information}

Supplementary information The online version contains supplementary material available at https://doi.org/10.1038/s41467-021-23349-9.

Correspondence and requests for materials should be addressed to G.W. or X.H.

Peer review information Nature Communications thanks the anonymous reviewer(s) for their contribution to the peer review of this work. Peer reviewer reports are available.

Reprints and permission information is available at http://www.nature.com/reprints

Publisher's note Springer Nature remains neutral with regard to jurisdictional claims in published maps and institutional affiliations.

Open Access This article is licensed under a Creative Commons Attribution 4.0 International License, which permits use, sharing, adaptation, distribution and reproduction in any medium or format, as long as you give appropriate credit to the original author(s) and the source, provide a link to the Creative Commons license, and indicate if changes were made. The images or other third party material in this article are included in the article's Creative Commons license, unless indicated otherwise in a credit line to the material. If material is not included in the article's Creative Commons license and your intended use is not permitted by statutory regulation or exceeds the permitted use, you will need to obtain permission directly from the copyright holder. To view a copy of this license, visit http://creativecommons.org/ licenses/by/4.0/.

(C) The Author(s) 2021 